\title{
Population-level asymmetry of the cerebral cortex: reproducibility, lifespan changes, heritability, and individual differences
}

\author{
Authors and affiliations \\ James M. Roe ${ }^{1 *}$, Didac Vidal-Piñeiro ${ }^{1}$, Inge K. Amlien ${ }^{1}$, Mengyu Pan ${ }^{1}$, Markus H. Sneve ${ }^{1}$, Michel Thiebaut \\ de Schotten ${ }^{2,3}$, Patrick Friedrich ${ }^{4}$, Zhiqiang Sha ${ }^{5}$, Clyde Francks ${ }^{5,6}$, Yunpeng Wang ${ }^{1}$, Kristine B. \\ Walhovd $^{1,7}$, Anders M. Fjell ${ }^{1,7}$ \& René Westerhausen ${ }^{8}$ \\ ${ }^{1}$ Center for Lifespan Changes in Brain and Cognition (LCBC), Department of Psychology, University of Oslo, Norway. \\ ${ }^{2}$ Groupe d'Imagerie Neurofonctionnelle, Institut des Maladies Neurodégénératives-UMR 5293, CNRS, CEA, University of \\ Bordeaux, Bordeaux, France. \\ ${ }^{3}$ Brain Connectivity and Behaviour Laboratory, Sorbonne Universities, Paris, France. \\ ${ }^{4}$ Institute of Neuroscience and Medicine (INM-7: Brain and Behaviour), Research Centre Jülich, Jülich, Germany \\ ${ }^{5}$ Language and Genetics Department, Max Planck Institute for Psycholinguistics, Nijmegen, The Netherlands. \\ ${ }^{6}$ Donders Institute Netherlands for Brain, Cognition and Behaviour, Radboud University, Nijmegen, The Netherlands. \\ ${ }^{7}$ Department of Radiology and Nuclear Medicine, Oslo University Hospital, Oslo, Norway. \\ ${ }^{8}$ Section for Cognitive and Clinical Neuroscience, Department of Psychology, University of Oslo, Norway.
}

*Address correspondence to James M. Roe, Department of Psychology, PO Box 1094 Blindern, 0317 Oslo, Norway.

Email: j.m.roe@psykologi.uio.no

Running title: Population-level cortical asymmetry

Keywords: Lateralization, population neuroscience, lifespan

Open access license: yes

The authors declare no competing interests

\begin{abstract}
Cortical asymmetry is a ubiquitous feature of brain organization that is altered in neurodevelopmental disorders and aging. Achieving consensus on cortical asymmetries in humans is necessary to uncover the genetic-developmental mechanisms that shape them and factors moderating cortical lateralization. Here, we delineate population-level asymmetry in cortical thickness and surface area vertex-wise in 7 datasets and chart asymmetry trajectories across life (4-89 years; observations $=3937 ; 70 \%$ longitudinal). We reveal asymmetry interrelationships, heritability, and test associations in UK Biobank $(\mathrm{N}=\sim 37,500)$. Cortical asymmetry was robust across datasets. Whereas areal asymmetry is predominantly stable across life, thickness asymmetry grows in development and declines in aging. Areal asymmetry correlates in specific regions, whereas thickness asymmetry is globally interrelated across cortex and suggests high directional variability in global thickness lateralization. Areal asymmetry is moderately heritable (max $h^{2}{ }_{S N P} \sim 19 \%$ ), and phenotypic correlations are reflected by high genetic correlations, whereas heritability of thickness asymmetry is low. Finally, we detected an asymmetry association with cognition and confirm recently-reported handedness links. Results suggest areal asymmetry is developmentally stable and arises in early life, whereas developmental changes in thickness asymmetry may lead to directional variability of global thickness lateralization. Our results bear enough reproducibility to serve as a standard for future brain asymmetry studies.
\end{abstract}

\section{Significance}

Cortical asymmetry is reduced in neurodevelopmental disorders, yet we lack knowledge of how cortical asymmetry development proceeds across life in health. We provide a definitive reference for asymmetry in the cerebral cortex. We find areal asymmetry is stable from childhood to old age, and specific areal asymmetries are formed under common genetic-developmental influence. In contrast, thickness asymmetry shows developmental growth, and is globally interrelated in a pattern suggesting highly left-lateralized individuals tend towards left-lateralization also in right-asymmetric regions (and vice versa). Heritability mapping also supported a prenatal-postnatal developmental dichotomy for areal and thickness asymmetry, and we find reduced asymmetry in the most lateralized brain region associates with reduced cognition. Our results provide novel insights into normal brain organization and development. 
bioRxiv preprint doi: https://doi.org/10.1101/2021.11.25.469988; this version posted December 8, 2021. The copyright holder for this preprint (which was not certified by peer review) is the author/funder, who has granted bioRxiv a license to display the preprint in perpetuity. It is made available under aCC-BY-NC 4.0 International license.

\section{Introduction}

The brain's hemispheres exhibit high contralateral symmetry ${ }^{1,2}$ and homotopic regions are amongst the most genetically ${ }^{3-5}$ and developmentally linked ${ }^{3,6}$. However, structural asymmetry is also a ubiquitous aspect of brain organization 7,8 . Cortical thickness (CT) and surface area (SA) are known to exhibit distinct asymmetry patterns ${ }^{7,9}$, but these have been reported inconsistently $7,8,10-20$. Yet disrupted cortical asymmetry is a confirmed feature of neurodevelopmental disorders 21 , aging ${ }^{10}$, and Alzheimer's disease ${ }^{10,22}$. Hence, achieving consensus on cortical asymmetries and understanding the genetic-developmental and lifespan influences that shape and alter them is necessary to discover precise biomarkers for disease. To reach consensus, an atlas-free description of asymmetries that reliably replicate across international samples (i.e. population-level asymmetries) is needed. This would enable precision mapping of the genetic and individual-specific factors moderating cortical lateralization, and serve as a high-fidelity phenotype for future studies on brain asymmetry. Furthermore, it is unknown how cortical asymmetry development proceeds across life, as no previous study has mapped cortical asymmetry trajectories longitudinally across the lifespan.

Although several studies have mapped cortical asymmetry ${ }^{7,8,10-20,23}$, conflicting results may be partly due to the use of brain atlases with varying spatial resolutions, especially if asymmetry conforms poorly to the predefined anatomical boundaries. Still, even amongst studies adopting an atlas-free approach, conflicting results abound ${ }^{13-18}$. For example, for CT asymmetry, medial prefrontal cortex (mPFC) has been reported to show both extensive rightward ${ }^{14,16,17}$ and leftward ${ }_{10-12,20}$ lateralization. Beyond regional inconsistencies, a recent meta-analysis confirmed the cortex is globally organized in a characteristic pattern of CT asymmetry, wherein anterior and posterior regions are thicker in the left and right hemisphere, respectively ${ }^{7}$. Notably, while this agrees with some reports ${ }^{10-12,20,24}$, it is less compatible with many others ${ }^{8,13-19,25}$. Furthermore, that study applied a relatively course brain atlas, and there is currently no high-resolution complement to describe cortical asymmetries that reliably reproduce across international samples (but see ${ }^{10,24}$ ). For areal asymmetry, while results have been broadly more consistent ${ }^{7,8,14,19,23,26}$, there nevertheless remain important discrepancies, such as reports of right- $7,27,28$ and left- ${ }^{14,26}$ lateralization of superior temporal sulcus (STS).

An accurate description of the lifespan trajectories of cortical asymmetry may shed light on mechanisms underlying diverse aspects of asymmetry across life. For CT, longitudinal increases in asymmetry have been shown during the first two years of life ${ }^{11}$, with suggestions of rapid asymmetry growth from birth to 1 year ${ }^{11}$, and continued growth until adolescence ${ }^{29}$. However, previous studies mapped CT asymmetry linearly across cross-sectional developmental and adult age-ranges 13,20 , mostly concluding CT asymmetry is minimal in infancy and maximal age $\sim 60$. In contrast, recent work established CT asymmetry shows a non-linear decline from 20 to 90 years that is reproducible across aging cohorts ${ }^{10}$. Thus, although offering viable developmental insights ${ }^{13,20}$, previous lifespan studies of CT asymmetry do not accurately capture the aging process, and likely conflate non-linear developmental and aging trajectories with linear models. A longitudinal exploration of the lifespan trajectories of CT asymmetry accounting for dynamic change is needed to further knowledge of normal brain development.

In addition, few studies have charted developmental ${ }^{26,28}$ or aging effects ${ }^{7}$ on SA asymmetry. However, indirect evidence suggests SA asymmetry may exhibit little change from birth to 2 years ${ }^{26}$ despite rapid and concurrent developmental cortical expansion ${ }^{30}$. Determining the developmental timing of cortical asymmetry will provide a useful normative reference, as subtly altered asymmetry in neurodevelopmental disorders suggests early life perturbations in left-right brain organization contribute to lifelong detriment in brain health ${ }^{21,31}$.

Correlations between cortical asymmetries in adults may provide a window on asymmetries formed under common geneticdevelopmental influence. Yet while there has been much research on whether asymmetries of various morphometric measures ${ }^{8,14,19}$ or imaging modalities ${ }^{27}$ relate to one another, few have focused on interrelationships between asymmetries derived from the same metric. Where reported, evidence suggests cortical asymmetries are mostly independent ${ }^{32,33}$ - in line with a multifactorial view of asymmetry phenotypes ${ }^{34-36}$ - and a recent study found asymmetry in anatomically connected regions of the cortical language network was no more related than in regions selected at random 27. Currently, it is not known whether or how cortical asymmetries correlate within individuals, though this may suggest coordinated development of left-right brain asymmetries.

Altered lateralization has been hypothesized to relate to poorer cognitive outcomes ${ }^{20,37,38}$. In line with this, recent work suggests genetic overlap between cortical asymmetry, educational attainment, and neurodevelopmental disorders ${ }^{31}$, and reduced brain torque $25,39,40$ - a gross morphological asymmetry with a strong population-level bias - associates with lower cognition ${ }^{41}$. For CT and SA asymmetry, however, reported asymmetry-cognition associations have been conflicting 20,42,43 and remain untested in large-scale data. Furthermore, most large-scale studies of the factors moderating cortical asymmetry have adopted brain atlases offering limited spatial precision $7,31,44$. Accordingly, previous large-scale studies did not detect associations with handedness ${ }^{7,45}$ that were not found until a recent study applied vertex-wise mapping in big data ${ }^{24}$. Similarly, it is unclear to what degree poor-fitting atlases drive down heritability estimates of cortical asymmetry 7,31 , as estimates improve when brain measures better conform to the biology under genetic investigation ${ }^{46,47}$. However, no previous study has assessed heritability after precisely delineating regions of cortex that are asymmetric at the population level, and cortex-wide heritability maps have all used the same atlas we propose fits poorly to the asymmetry of cortex ${ }^{48}$.

Here, we 1) delineate population-level cortical SA and CT asymmetries using vertex-wise analyses and their overlap in 7 international datasets, and 2) map their trajectories longitudinally across the lifespan. We 3) investigate interregional asymmetry correlations, asking whether and how asymmetries correlate within individuals. Next, we 4) tested heritability 
bioRxiv preprint doi: https://doi.org/10.1101/2021.11.25.469988; this version posted December 8,2021 . The copyright holder for this preprint (which was not certified by peer review) is the author/funder, who has granted bioRxiv a license to display the preprint in perpetuity. It is made available under aCC-BY-NC 4.0 International license.

of cortical asymmetry using both an extended twin design and genome-wide single nucleotide polymorphism (SNP) data. Finally, we 5) screened our set of robust, population-level asymmetries for association with general cognitive ability, handedness, sex, and brain size in UK Biobank (UKB) ${ }^{49}$.

\section{Results}

\subsection{Population-level asymmetry of the cerebral cortex}

First, to delineate cortical regions exhibiting population-level SA and CT asymmetry, we assessed asymmetry vertex-wise in 7 independent samples and quantified overlapping effects (Methods). SA asymmetries were markedly consistent across all 7 datasets (Fig. 1A): the spatial overlap between Al maps ranged from $r=.88$ to .97 (Fig. 1C). Across all 7 datasets (Fig. 1D), strong leftward SA asymmetry was observed in a large cluster in supramarginal gyrus (SMG) that spanned the length of postcentral gyrus, extended inferiorly into planum temporale and primary auditory regions and conformed markedly to their anatomical boundaries (see SI Fig.1A for significance). We also observed consistently strong leftward asymmetry in anterior insula, anterior temporal cortex, rostral anterior cingulate, medially in superior frontal cortex, and precuneus, the latter extending the length of parahippocampal gyrus into entorhinal cortex. Strong rightward SA asymmetry was consistently evident in cingulate cortex, inferior parietal cortex, STS, lateral and medial occipital cortex, and in mPFC and rostral middle frontal cortex (Fig. 1A). The global pattern agrees with previous reports ${ }^{7,23,24}$, and effects showed markedly high overlap across datasets (Fig. 1D)

For CT, an anterior-posterior pattern of left-right asymmetry was evident in most datasets (Fig. 1B), consistent with recent reports ${ }^{7,10,24}$. Though spatial correlations between $\mathrm{Al}$ maps were high, they were notably more variable $(r=.33-.93$; Fig. 1C); HCP showed lower correlation with all datasets $(r=.33-.46)$ whereas all other datasets correlated highly with each other ( $\min r=.78$ ). Strong leftward CT asymmetry was evident in cingulate cortex, postcentral gyrus, and in superior frontal cortex - with consistent effects across datasets (Fig. 1E) - and in medial and lateral prefrontal cortex, though the latter two were less consistent among datasets. Strong rightward CT asymmetry was consistently evident in a large cluster in and around STS and lateral temporal cortex (Fig 1E; SI Fig 1B), insula, lingual gyrus, anterior parahippocampal and entorhinal cortex. Both SA and CT asymmetry extended beyond these described effects (SI Figs. 1-2).

Based on effect size criteria (Fig. 1D-E; Methods), we derived a set of robust clusters exhibiting population-level asymmetry for SA (14 clusters) and CT (20 clusters) to be used in further analyses (see SI Tables 5-6 for anatomical descriptions, see SI Fig 3 for variances). We then formally compared our approach to asymmetry estimates derived from a gyral-based atlas often used to assess asymmetry ${ }^{7,19,31}$, finding fairly poor correspondence with the vertex-wise structure of cortical asymmetry, particularly for CT (see SI; SI Fig. 4).

A

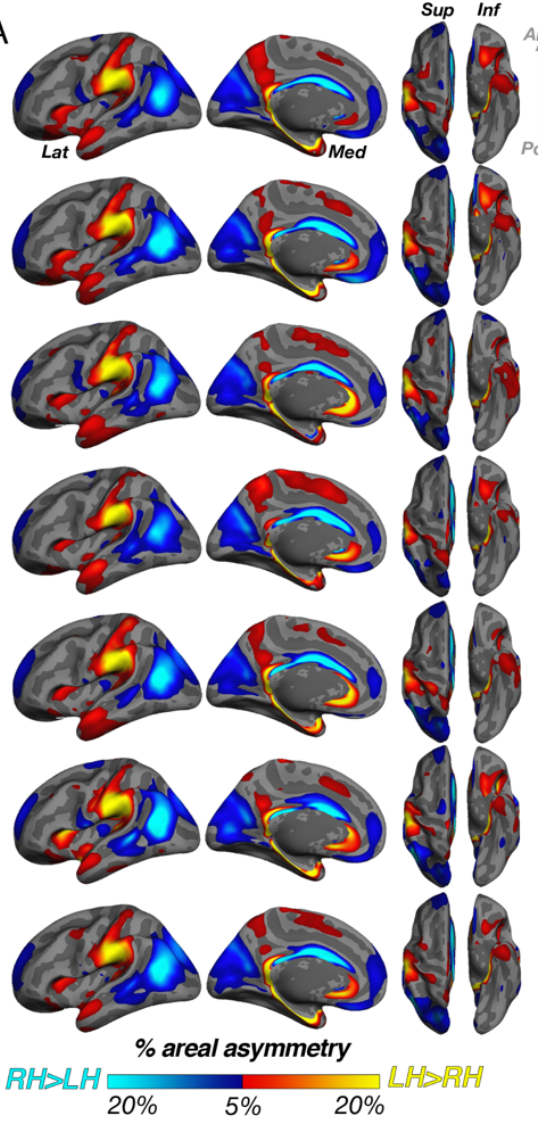

$\angle C B C$

Cam-CAN

DLBS

B
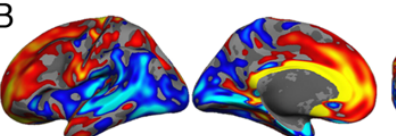

SALD
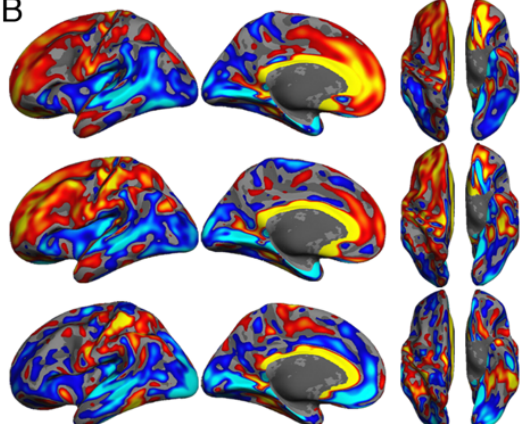

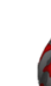

IXI
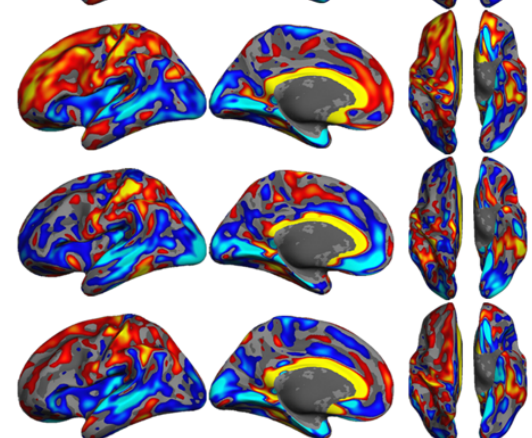

HCP
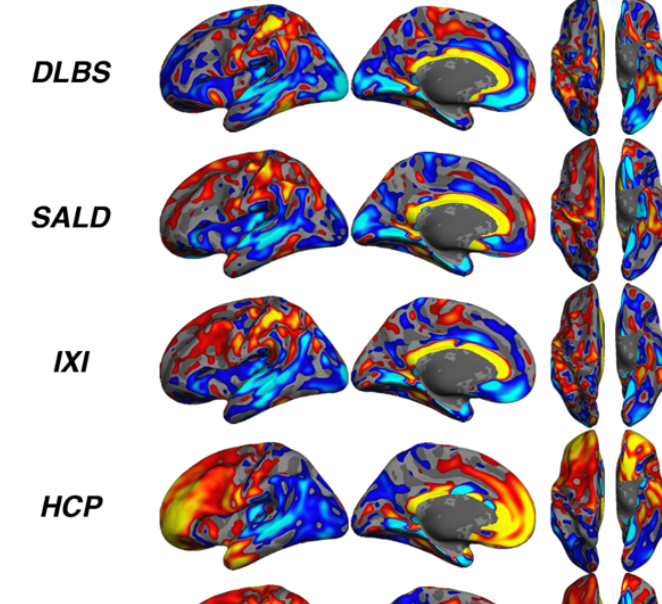

UK

Biobank

$(N=1000)$

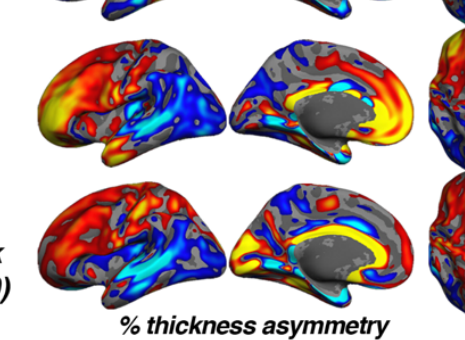

RH $>$ LH $5 \%$ 1\%
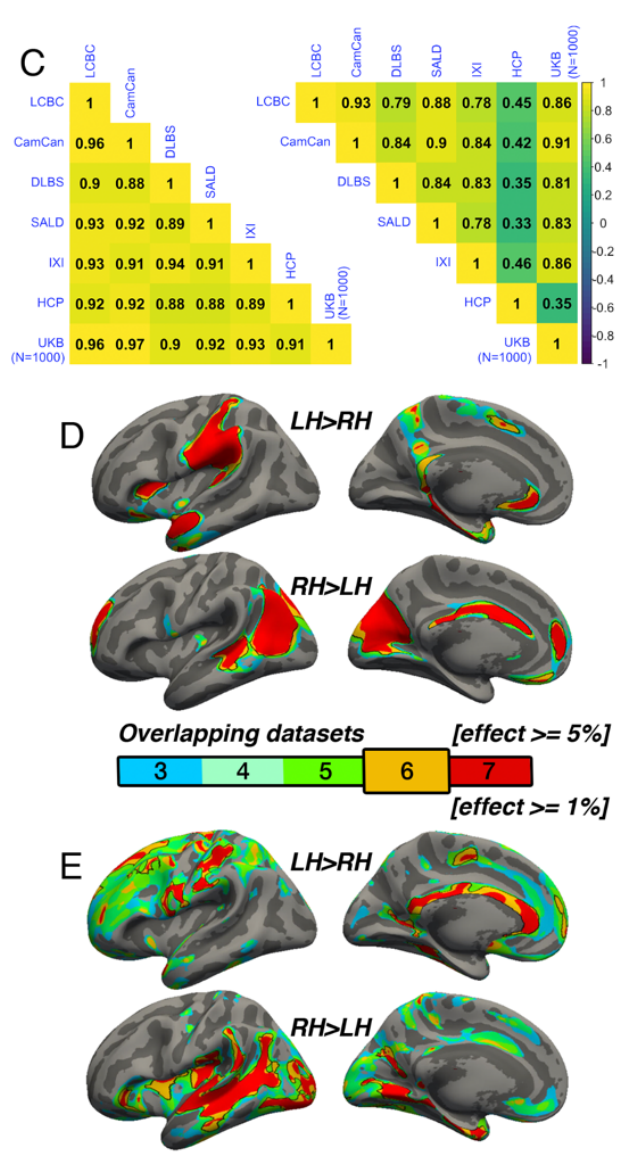
[effect $>=1 \%$ ]

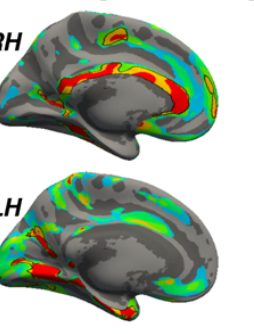


bioRxiv preprint doi: https://doi.org/10.1101/2021.11.25.469988; this version posted December 8, 2021. The copyright holder for this preprint (which was not certified by peer review) is the author/funder, who has granted bioRxiv a license to display the preprint in perpetuity. It is made available under aCC-BY-NC 4.0 International license.

Figure 1. A) Mean SA and B) CT asymmetry in each dataset. Warm and cold colours depict leftward and rightward asymmetry, respectively. C) Spatial overlap (Pearson's $r$ ) of the unthresholded maps between datasets for SA (lower matrix) and CT asymmetry (upper). D) Overlapping effects across datasets were used to delineate clusters exhibiting population-level SA (lower threshold $=5 \%$ ) and E) CT asymmetry (lower threshold $=1 \%$ ) based on a minimum 6-dataset overlap (black outlined clusters). Post=posterior; Lat=lateral; Med=medial; Ant=anterior; Sup=superior; Inf=inferior.

\subsection{Lifespan trajectories of cortical asymmetry}

We have recently shown that cortical regions exhibiting age-related reduction of CT asymmetry correspond with regions exhibiting strong asymmetry on average ${ }^{10}$. Thus, having delineated regions exhibiting population-level SA and CT asymmetry, we aimed to characterize the trajectories of SA and CT asymmetries longitudinally across the lifespan (4-89 years). To account for potentially non-linear trajectories, we used Generalized Additive Mixed Models (GAMMs) that enabled modelling the smooth left- $(\mathrm{LH})$ and right hemisphere $(\mathrm{RH})$ age-trajectories within our robust clusters (Methods).

In all clusters, SA asymmetry was strongly established already by age $\sim 4$ years (SI Fig. 5-6), and the lifespan trajectories of both leftward (Fig. 2A) and rightward (Fig. 2B) SA asymmetries were largely parallel. Specifically, a large left-asymmetric region in and around SMG/perisylvian (\#1; Fig. 2A) showed strong asymmetry by age $\sim 4$ that was maintained throughout life through steady aging-associated decline of both hemispheres, whereas leftward asymmetry of temporal cortex $(\# 2,6)$ and anterior insular (\#4) was maintained through developmental expansion and aging-associated decline of both hemispheres. Others (retrosplenial \#5; mPFC \#3,7) showed growth from pre-established asymmetry and more variable lifespan trajectories. On the other side, rightward asymmetries showed largely preserved asymmetry through agingassociated decline of both hemispheres (Fig 2B; medial occipital \#1; lateral parietal \#2; STS \#5; orbitofrontal \#7), through bilateral developmental expansion and aging-associated decline (mPFC \#6), or steadily expanding bilateral SA until midlife (cingulate; \#3). Though asymmetry trajectories did show significant change at some point throughout life in most clusters (SI Table 5), factor-smooth GAMM interaction analyses (SI Figs. 5-6) confirmed that asymmetry was significantly different from 0 across the entire lifespan in all SA clusters, and the average trajectories across all leftward and rightward clusters were clearly parallel (though still exhibited a significant difference; bordered plots in Fig. 2A-B; SI Table 5).

In contrast, though homotopic trajectories of CT clusters were more variable, they were mostly characterized by developmental increase and aging-associated decrease in asymmetry (i.e. non-parallel lifespan trajectories), through unequal rates of continuous thinning between the hemispheres from age $\sim 4$ (Fig. 2C-D; see SI Fig. 5B). Specifically, leftward CT asymmetry developed through comparatively slower thinning trajectories of the LH, whereas rightward asymmetry developed through slower RH thinning. In general, asymmetry development was evident up to a peak around age 25 for both leftward (Fig. 2C; superior frontal \#2; precentral \#4, frontal \#8,9,10; calcarine \#11) and rightward clusters (Fig 2D; \#1-9) and declined thereafter (see also SI Fig. 7). Factor-smooth GAMMs (SI Figs. 5-6) confirmed that the developmental foundation for CT asymmetry was already established by age $\sim 4(95 \%$ of CT clusters exhibited small but significant asymmetry at age $\sim 4$; SI Fig. $6 \mathrm{~B}$ ), and again asymmetry trajectories showed significant change at some point throughout life (SI Table 6). The average trajectories across all leftward and rightward clusters showed developmental asymmetry increase up to age $\sim 25$ and aging-associated asymmetry decrease from mid to old age (bordered plots; Fig 3). Results were robust to varying the number of knots used to estimate trajectories (see SI Fig. 5). 
bioRxiv preprint doi: https://doi.org/10.1101/2021.11.25.469988; this version posted December 8, 2021. The copyright holder for this
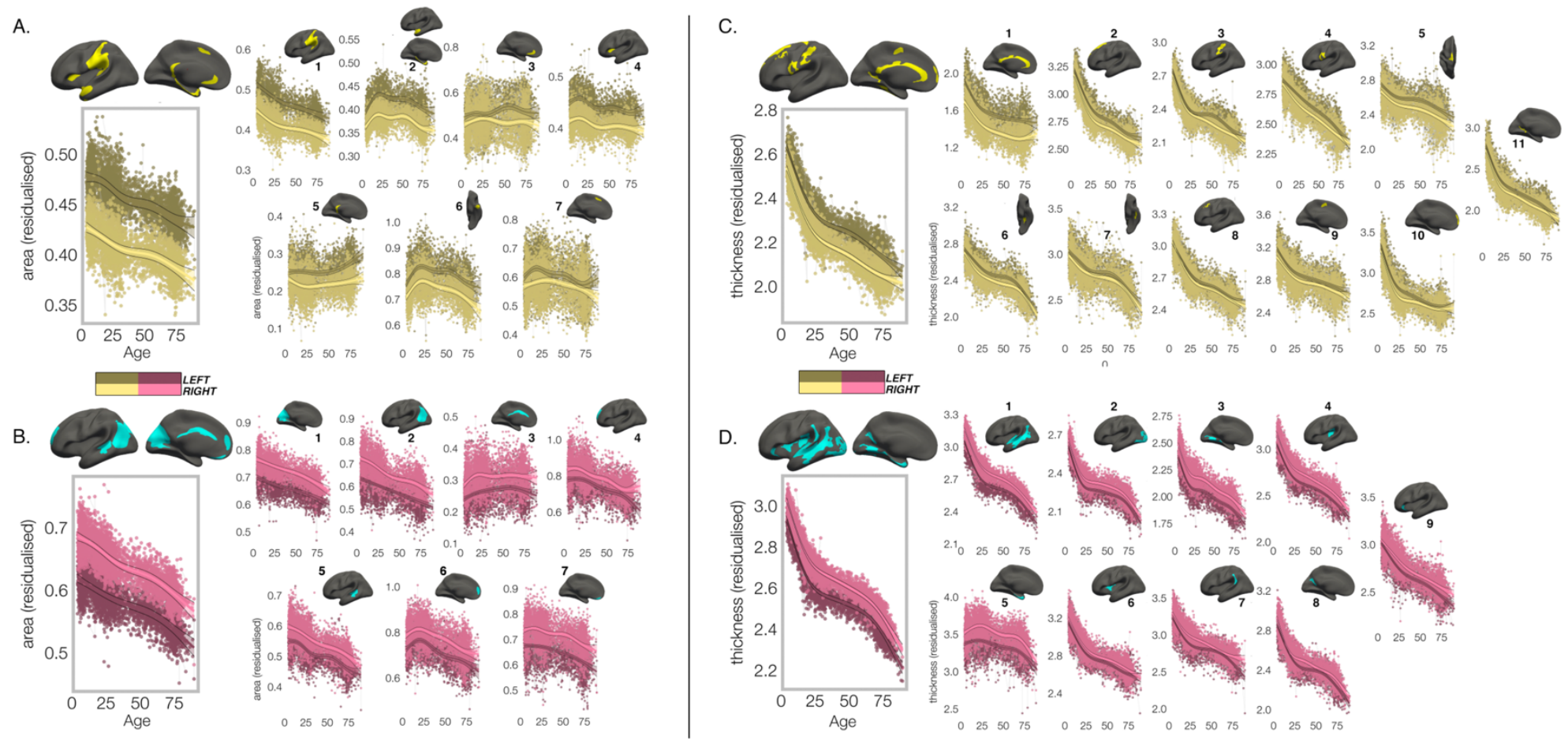

Figure 2: Homotopic lifespan trajectories in clusters exhibiting population-level a) leftward (yellow plots; yellow clusters) and b) rightward (pink plots; blue clusters) SA asymmetry ( $\mathrm{mm}^{2}$; average area of a vertex), and those exhibiting c) leftward and d) rightward CT asymmetry. Larger plots show the mean age trajectory across all clusters exhibiting leftward (top) and rightward (bottom) SA (left) and CT asymmetry (right). Dark colours correspond to LH trajectories. Data is residualized for sex, scanner and random subject intercepts. Clusters numbered for reference.

\subsection{Interregional asymmetry correlations}

We then investigated whether cortical asymmetry correlates within individuals (Methods).

For SA, a common covariance structure between asymmetries was detectable across datasets: LCBC, UKB and HCP all correlated almost perfectly ( $r>=0.97$, all $p<10^{-5}$; Fig. 3A; SI Fig. 9). The highest correlations (or "hotspots") all reflected positive correlations between regions that are on average left-asymmetric and regions that are on average right-asymmetric (i.e. higher leftward asymmetry in one region related to higher rightward asymmetry in another; Fig $3 \mathrm{~A}$ black outline); leftward asymmetry in SMG/perisylvian (\#1L) was related to higher rightward asymmetry in inferior parietal cortex (\#2R; $r$ $=.46[\mathrm{LCBC}]$ ), leftward anterior cingulate asymmetry (ACC; \#3L) was related to higher rightward asymmetry in mPFC (\#6R, $r=.46)$, and leftward asymmetry in a superior frontal cluster (\#7L) was related to rightward asymmetry in the cingulate (\#3R, $r=.67)$. None of the relationships could be explained by brain size, as additionally removing the effect of intracranial volume (ICV) from cluster Al's had a negligible effect on their interrelations (max correlation change $=0.008$ ). Post-hoc tests confirmed that opposite-direction asymmetries were more correlated if closer in cortex (SI Methods); geodesic distance was lower between cluster-pairs that were more correlated (rho = -.37 [LCBC]; -.38 [UKB; Fig. 3C]; -.32 [HCP]; all $p<.02$ ), though this was driven by the aforementioned "hotspots". By contrast, same-direction SA asymmetries were not more correlated if closer in cortex (leftward [all $p>.5$ ]; rightward [all $p>.5$ ]). This suggests specific SA asymmetries that are closer in cortex and opposite in direction may show coordinated development.

For CT asymmetry, the correlation matrix exhibited a clear pattern in UKB that was less visible but still apparent in LCBC and HCP (Fig. 3B). Mantel tests confirmed that the covariance structure replicated between all dataset-pairs (LCBC-HCP $r=.45, p=.006$; LCBC-UKB $r=.43, p=.01$; UKB-HCP $r=.46, p=.01$ ). The observed pattern suggested higher leftward asymmetry in regions that are on average left-asymmetric was associated with less rightward asymmetry in regions that are on average right-asymmetric. However, given that the Al measure is bidirectional, closer inspection of the correlations revealed that higher leftward asymmetry in regions that are left-asymmetric actually corresponded to more leftward asymmetry in right-asymmetric regions, and vice versa (and on average; see SI Fig. 10). In other words, individuals may tend towards either leftward lateralization or rightward lateralization (or symmetry) on average, irrespective of the regionspecific direction of mean asymmetry in the cluster. Similarly, asymmetry in left-asymmetric regions was mostly positively correlated, and asymmetry in right-asymmetric regions was mostly positively correlated. Again, additionally removing ICVassociated variance had negligible effect. Post-hoc principal components analysis (PCA) in UKB revealed PC1 explained $21.9 \%$ of the variance in CT asymmetry and strongly suggested a single global factor for CT asymmetry (Fig. 3D). Accordingly, we found a strong correlation between mean asymmetry across all leftward vs. mean asymmetry across all 
bioRxiv preprint doi: https://doi.org/10.1101/2021.11.25.469988; this version posted December 8,2021 . The copyright holder for this preprint (which was not certified by peer review) is the author/funder, who has granted bioRxiv a license to display the preprint in perpetuity. It is made available under aCC-BY-NC 4.0 International license.

rightward clusters in UKB $\left(r=.61 ; p<10^{-16}\right.$; Fig. 3D). Though less strong, relationships were significant in LCBC $(r=-.10$; $\left.p=1.3^{-4}\right)$ and $\mathrm{HCP}\left(r=-.10 ; p=1.7^{-4}\right.$; SI Fig. 11). Opposite-direction CT asymmetries that were closer in cortex were more negatively correlated in LCBC $(r h o=.29, p=.004)$ but not HCP $(p=.33)$ or UKB $(p=.84)$, whereas CT asymmetry in leftasymmetric $(r h o=-.44$ [LCBC]; rho $=-.44$ [UKB], rho $=-.29[\mathrm{HCP}]$, all $p<.05)$ and right-asymmetric $(r h o=-.34[\mathrm{LCBC}]$; rho $=-.49$ [UKB], rho $=-.57$ [HCP]; all $p<.05)$ regions was more positively correlated in cluster-pairs that were closer in cortex. These results suggest CT asymmetry is globally interrelated across the cortex and shows high directional variability in the adult population.

A
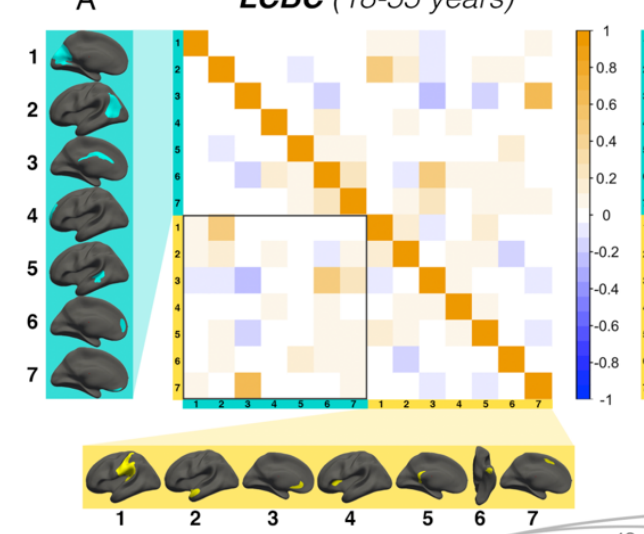

$\mathrm{B}$
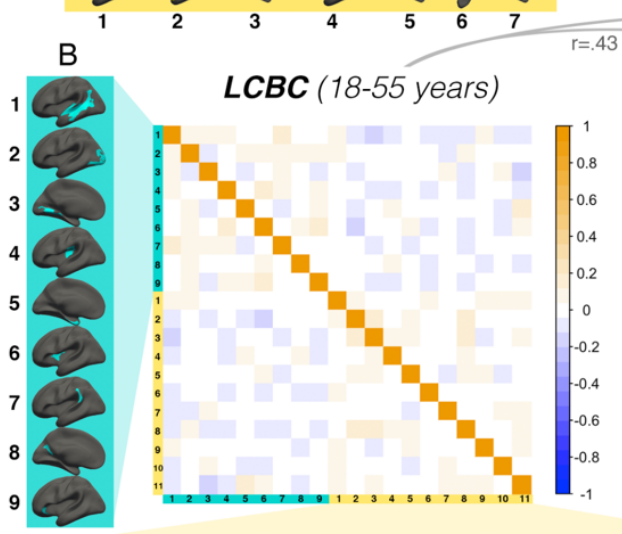

LCBC (18-55 years)

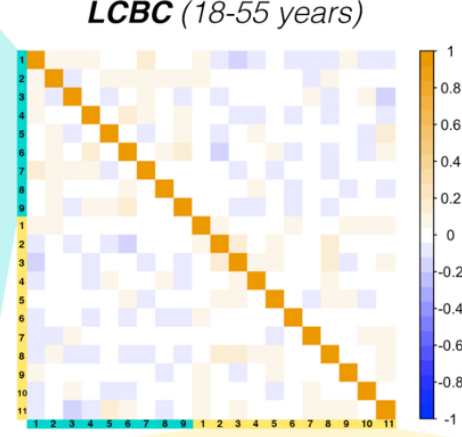

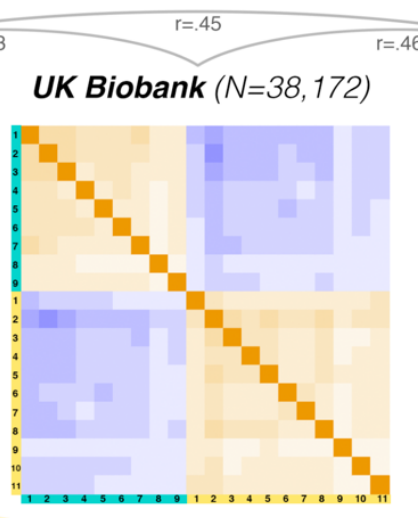
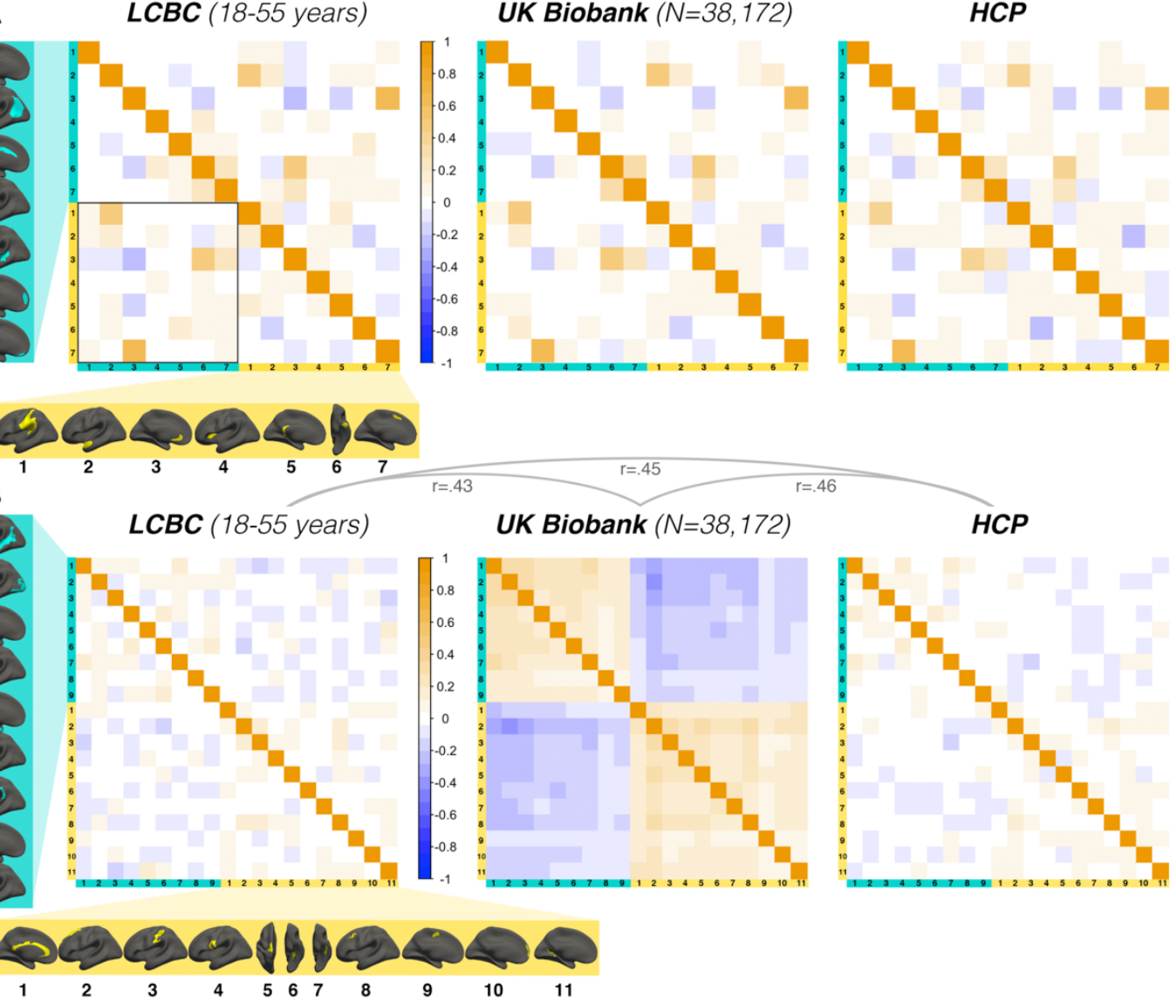

$10 \quad 11$

Figure 3: Interregional correlations between A) SA asymmetries and B) CT asymmetries for each replication dataset (Al's residualized for age, sex, scanner). Al's in rightward clusters are inversed, such that positive correlations denote positive asymmetry-asymmetry relationships regardless of direction. Yellow and blue brain clusters/colours denote leftward and rightward asymmetries, respectively (clusters numbered for reference). A consistent covariance structure was evident both for $S A(r>=.97)$ and CT asymmetry ( $r>=.43$; results above matrices). Black box in A highlights relationships between opposite-direction asymmetries (i.e. leftward vs rightward regions). C) For SA, opposite-direction cluster-pairs that were closer in cortex were more positively correlated (datapoints show cluster-pairs). D) A single component explained $21.9 \%$ variance in CT asymmetry in UKB (inset plot). Accordingly, we found a strong correlation $\left(r=-.61 ; p<10^{-16}\right)$ in UKB between mean asymmetry across leftward clusters ( $Y$-axis) vs. mean asymmetry across rightward clusters ( $X$-axis; Al's in rightward clusters inversed). Lines of symmetry (0) are in dotted grey (see also SI Figs. 9-11).

\subsection{Heritability}

Heritability of global AI measures was low and only significant for SA asymmetry in UKB (SA $h^{2}{ }_{S N P}=.07 ; p=2.16 e^{4} ;$ CT $\mathrm{h}^{2}$ SNP $=.01 ; p=.22$, SI Table 7). For SA, only two clusters showed significant heritability in HCP and these did not survive multiple comparison correction. In contrast, SNP-based analyses revealed 71\% (10/14) of SA asymmetry clusters exhibited significant heritability (see SI Table 8). Importantly, highest heritability was observed for leftward SA in the anterior insula cluster $\left(\mathrm{h}^{2} \mathrm{SNP}=18.6 \%, p<10^{-10}\right.$; SI Table 8$)$, which was substantially higher than the next highest estimates in SMG/perisylvian $\left(h^{2}{ }_{S N P}=10.7 \%, p=3.01 \mathrm{e}^{-9}\right)$, retrosplenial cortex, gyrus rectus and the cingulate (all $\left.\mathrm{h}^{2} \mathrm{SNP}=8-10 \%\right)$. For CT, no cluster survived in HCP, only $3 / 20(15 \%)$ clusters exhibited significant SNP-heritability, and estimates were lower $\left(h^{2}\right.$ SNP $=3-7 \%$; SI Table 9).

We then estimated cortex-wide heritability using a fine-grained parcellation ${ }^{50}$ (Fig. 4). For SA, though no parcels survived correction in HCP, $53 \%$ (267/500) of parcels exhibited significant SNP-heritability (post-correction) in UKB (p[FDR]<.05; 
bioRxiv preprint doi: https://doi.org/10.1101/2021.11.25.469988; this version posted December 8,2021 . The copyright holder for this preprint (which was not certified by peer review) is the author/funder, who has granted bioRxiv a license to display the preprint in perpetuity. It is made available under aCC-BY-NC 4.0 International license.

black outlines in Fig. 5A; parcels with suggestive significance in HCP [also in black outline] survived correction in UKB). Beyond significance, a consistent heritability pattern was clearly evident in both samples, notably in anterior insula, SMG, Sylvian fissure, STS, calcarine sulcus, cingulate, medial and orbitofrontal cortex, and fusiform (spatial correlation between maps; $r=.38 ; p<10^{-16}$ ). Importantly, maximum SNP-heritability (yellow parcel in Fig. 5) was observed in anterior insula (parcel $\mathrm{h}^{2}$ SNP $=16.4 \% ; p<10^{-10}$ ), confirming this region constitutes the most heritable cortical asymmetry in humans (and not improving on the cluster-wise estimate). For CT, we observed little overlap in heritability estimates between datasets (spatial correlation was significant but low; $r=.12 ; p=.01$ ). Significant FDR-corrected SNP-heritability was nevertheless observed around superior temporal gyrus, planum temporale, the posterior insula/Sylvian fissure, anterior insula, and in orbitofrontal cortex $\left(\max \mathrm{h}^{2} \mathrm{SNP}=16.6 \%\right)$, along the cingulate and in medial visual cortex. However, SNP-heritability for CT asymmetry was substantially lower $\left(\beta=-0.71, p<2 \mathrm{e}^{-16}\right)$, and higher estimates pertained to regions that were limited in extent but showed no clear global pattern.

For SA, large genetic correlations explained several phenotypic correlations in Fig. 3A (Fig. 4C): high genetic correlations were found between leftward asymmetry in SMG/perisylvian and higher rightward asymmetry in lateral parietal cortex $\left(\mathrm{LPC} ; r G=.83 ; p(F D R)=7.76 \mathrm{e}^{-05}\right)$, between leftward superior frontal cortex asymmetry and rightward asymmetry along the cingulate $\left(r G=.82 ; p[F D R]=1.36 \mathrm{e}^{-02}\right)$, and between leftward anterior temporal/parahippocampal asymmetry and rightward asymmetry in LPC $\left(r G=.68 ; p[F D R]=1.36 \mathrm{e}^{-02}\right)$. Genetic correlations between anterior insula and two rightward superior frontal clusters were also observed $\left(r G=.86 ; p[F D R]=1.41 \mathrm{e}^{-06} ; r G=0.42 ; p[F D R]=7.74 \mathrm{e}^{-04}\right)$ in the absence of phenotypic correlations (Fig. 3), and several same-direction asymmetries showed moderate genetic correlation. For CT, one cluster-pair survived FDR-correction (see Fig. $4 \mathrm{C} ; r G=0.68 ; p[F D R]=3.03 \mathrm{e}^{-02}$ ).
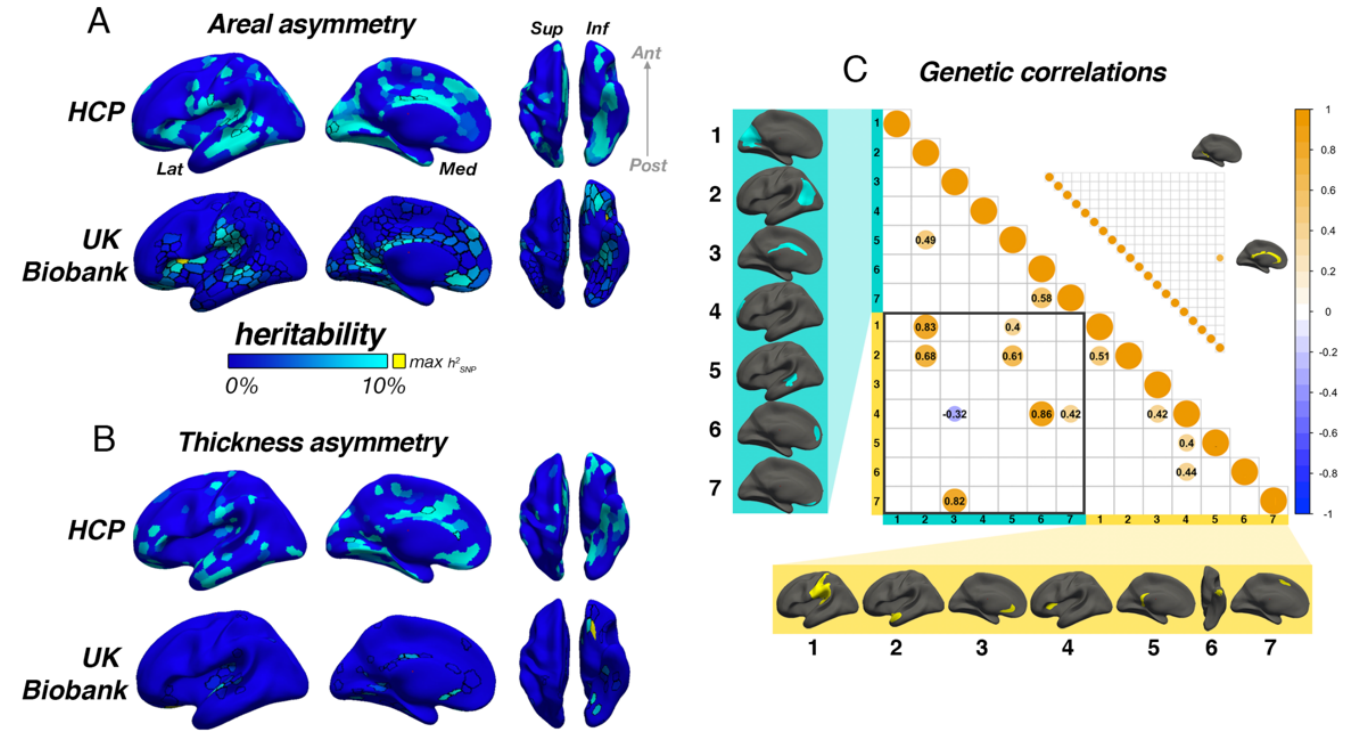

Figure 4. Heritability of $S A(\boldsymbol{A})$ and $C T$ asymmetry (B) estimated cortex-wide in HCP (top row) and UKB (bottom). Unthresholded effect maps are shown. In HCP, no parcel survived FDR-correction, whereas for UKB, 53\% of SA and $11 \%$ of CT parcels survived. Parcels in black outline show significance at $p<.05$ (uncorrected) for HCP, and at $p[F D R]<.05$ for UKB. Yellow parcels depict maximum SNP-heritability $\left(S A h^{2}=16.4 \%\right.$; CT $\left.h^{2}=16.6 \%\right)$. C) SNP-based genetic correlations between SA (lower matrix) and CT asymmetries (upper). For SA, genetic correlations explained several phenotypic correlations (Fig. 3A). For CT, one pair survived FDR-correction (shown). Al's in rightward clusters were inversed such that positive genetic correlations denote asymmetry-asymmetry genetic relationships regardless of direction. Yellow and blue brain clusters/colours denote population-level leftward and rightward asymmetries, respectively (clusters numbered for reference).

\subsection{Associations with Cognition, Handedness, Sex, and ICV}

Several significant associations were observed between factors-of-interest and asymmetry in our clusters (Fig. 5). Notably, all effect sizes were small. For general cognitive ability, we found one association: higher SA asymmetry in the largest leftward cluster (SMG/perisylvian) was significantly associated with better cognition $\left(\beta=.03[\mathrm{Cl}=0.02-0.04], p=4.1 \mathrm{e}^{-7}\right)$. This was checked in the substantially reduced non-imputed subset of data with no missing cognitive variables and retained the lowest $p$-value $\left(\mathrm{N}=4696 ; \beta=0.04[\mathrm{Cl}=0.01-0.07] ; p=6.9 \mathrm{e}^{-3}\right)$. For handedness, reduced leftward SA asymmetry in anterior insula and CT asymmetry along postcentral gyrus was found in left-handers, in line with our recent vertex-wise mapping in UKB ${ }^{24}$. For sex effects, which were also small, males typically exhibited slightly stronger SA asymmetry in large clusters (e.g. leftward SMG/perisylvian and temporal pole; rightward inferior parietal and superior frontal) but reduced leftward and rightward asymmetry in mPFC. For CT, males exhibited more rightward asymmetry in STS and posterior insula, more leftward CT asymmetry in superior frontal cortex, but reduced rightward CT asymmetry in entorhinal cortex and anterior insula, and reduced leftward asymmetry in caudal superior frontal cortex. As ICV effects were typically most nominal, these are shown in SI Fig. 12 (SI Tables 10-12). 
bioRxiv preprint doi: https://doi.org/10.1101/2021.11.25.469988; this version posted December 8, 2021. The copyright holder for this preprint (which was not certified by peer review) is the author/funder, who has granted bioRxiv a license to display the preprint in perpetuity. It is made available under aCC-BY-NC 4.0 International license.

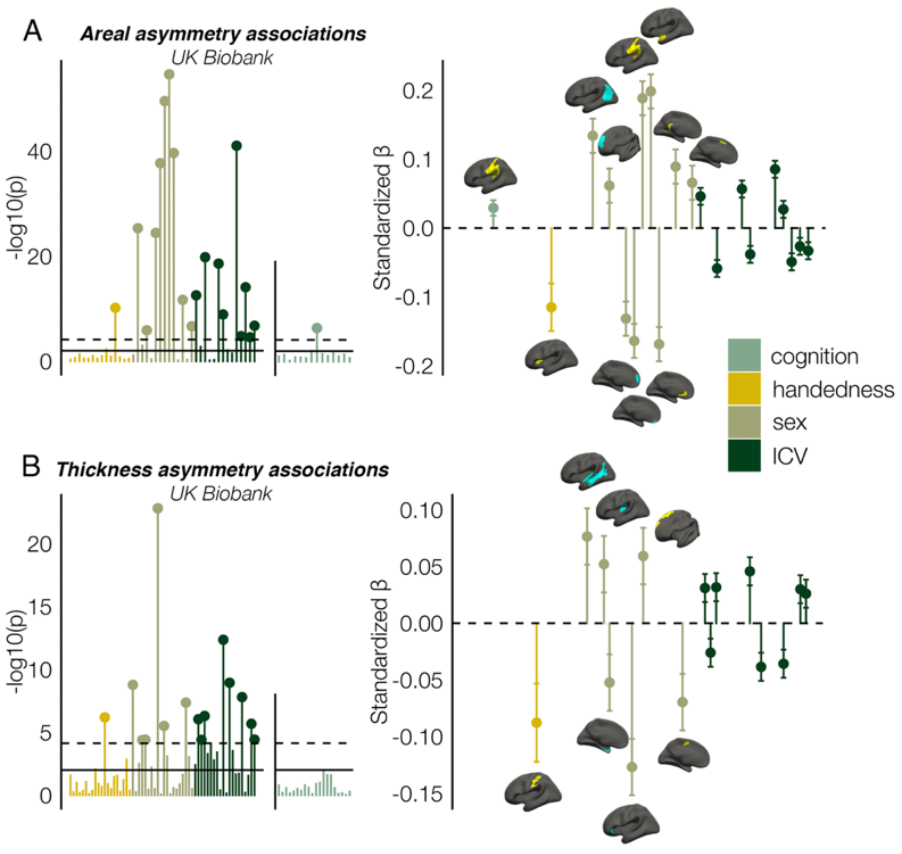

Figure 5. Associations with general cognitive ability (first principal component), Handedness, Sex, and estimated intracranial volume (ICV) in UKB, in clusters exhibiting population-level A) SA and B) CT asymmetry. Left plots denote significance (negative logarithm; corrected $\left[p<7.3 e^{-5}\right]$ and uncorrected threshold $[p=.01]$ shown by dotted and non-dotted line, respectively). X-axis displays the test for each cluster-association. As maximum sample size was used to test each association (Handedness, Sex and ICV: N=37,570), effects on cognition were tested in separate models with fewer observations ( $N=35,199$; separated association vvplots). Right plots denote effect sizes, 95\% confidence intervals (error bars) and cortical location of associations surpassing Bonferroni-corrected significance. Right handers and females are coded 0 , such that a negative effect for handedness / sex / ICV / cognition denotes less asymmetry in left handers / males / larger brains / higher cognition. Associations with ICV are shown in SI Fig. 12. Yellow and blue clusters denote leftward and rightward asymmetries, respectively.

\section{Discussion}

We provide a reference for population-level cortical asymmetries using 7 international datasets and offer the first description of the longitudinal lifespan trajectories of cortical asymmetry. Our results demonstrate the replicable interregional relationships between asymmetries within individuals, provide the most detailed heritability maps for cortical asymmetry to date, uncover novel and confirm previously-reported associations with factors reportedly related to asymmetry, and further knowledge on normal brain development. All maps are available at neurovault.org/XXXX.

Our vertex-wise description of cortical asymmetries that reproduce across cohorts replicates and completes a recent lowresolution meta-analysis ${ }^{7}$, and can serve as a high-fidelity phenotype for future brain asymmetry studies. The marked consistency across samples here suggests consensus may now be reached regarding cortical asymmetry phenotypes in humans, as our results agree with most of the literature $7,8,14,19,23,26,28$, including a recent large-scale mapping in mid-old age ${ }^{24}$. This consensus, along with the genetic findings presented herein, suggests genetic-developmental programs regulate mean brain lateralization with respect to SA, and the trajectories observed here suggest this form of cerebral asymmetry is maintained throughout life and formed early on - likely in utero ${ }^{26,31}$. For CT asymmetry - for which findings have been particularly mixed ${ }^{7,8,18-20,10-17}$ - the left-right patterning observed here is compatible with recent reports ${ }^{10,24}$, studies examining CT asymmetry from birth ${ }^{11}$, global meta-analyse ${ }^{7}$, reports using alternative analysis streams ${ }^{11,20}$, anatomical asymmetries evident early in ontogeny ${ }^{51,52}$, and leftward CT asymmetry overlapping language- ${ }^{53}$ and motorrelated regions ${ }^{54,55}$. This consistency across adult samples may also indicate that mean CT asymmetry is genetically regulated at the population-level in humans. However, our findings of development and decline of CT asymmetry across life ${ }^{10}$, higher directional variability in adult samples and lower heritability converge to suggest CT asymmetry may be more prone to lifespan change, potentially more malleable to life experience, and susceptible to lifespan accumulation of insult. Though it remains possible CT asymmetry change could be genetically regulated into old age, this interpretation agrees with work suggesting SA may trace to prenatal factors ${ }^{56,57}$ whereas CT relates more to postnatal lifespan influences ${ }^{57,58}$.

Conceivable sources of previously inconsistent results may be the age-distribution under study ${ }^{10}$ and the existence of varying directional asymmetries within the population (for CT asymmetry). This may partly explain why CT asymmetry metrics are more variable across datasets compared with SA ${ }^{7}$, though CT asymmetry effects are also smaller $8,14,19$ and likely contain more measurement error. Varying directional asymmetry within atlas-based parcels may also explain 
bioRxiv preprint doi: https://doi.org/10.1101/2021.11.25.469988; this version posted December 8, 2021. The copyright holder for this preprint (which was not certified by peer review) is the author/funder, who has granted bioRxiv a license to display the preprint in perpetuity. It is made available under aCC-BY-NC 4.0 International license.

inconsistent reports, such as in insular cortex where we observed consistent but discrepant asymmetry to that reported in ENIGMA ${ }^{7}$. However, this does not account for the discrepancy that studies using the same atlas ${ }^{48}$ typically report areal asymmetry in STS to be left-lateralized ${ }^{7,27,28}$, as the right-lateralization evidenced here and elsewhere ${ }^{14,26}$ seems unambiguous. And although we did not find strong SA asymmetry in inferior frontal regions as reported by Kong et al ${ }^{7}$, the unthresholded significance maps were somewhat compatible with this (SI Fig. 2). The high overlap in effects between 7 datasets from 4 countries suggests our results likely apply universally, though future studies will be needed to confirm this in non-American/North European samples.

Our longitudinal description of cortical asymmetry lifespan trajectories gleaned novel insight into normal brain development. For SA, adult-patterns of lateralization were strongly established already before $\sim 4$ years, indicating SA asymmetry traces back further and does not primarily emerge through later cortical expansion ${ }^{59}$. Rather, the lifespan trajectories predominantly show stability from childhood to old age, as SA asymmetry was generally maintained through periods of developmental expansion and aging-associated change that were region-specific and bilateral. This agrees with evidence indicating SA asymmetry is primarily determined in utero ${ }^{26}$, and indirect evidence suggesting little change in SA asymmetry from birth to 2 years despite rapid and concurrent cortical expansion ${ }^{26,30,59}$. It may also fit with the principle that the primary microstructural basis of SA 60 - the number of and spacing between cortical minicolumns - is determined in prenatal life ${ }^{58,60}$, and agree with evidence suggesting asymmetry at this microstructural level may underly hemispheric differences in $\mathrm{SA}^{61}$. The developmental trajectories agree with studies indicating SA asymmetry is established and strongly directional early in life ${ }^{26,28}$. That anatomical change in later development specifically in SA follows embryonic gene expression gradients may also agree with a prenatal account for SA asymmetry ${ }^{58}$. These results may therefore constrain the extent to which SA asymmetry can be viewed as a plastic feature of brain organization, and may even suggest SA asymmetry may sometimes be a marker for innate hemispheric specializations shared by most humans. The high degree of precision with which leftward SA asymmetry follows the contours of auditory-related regions in the Sylvian fissure (SI Fig. 1A) which show left functional lateralization in humans may be one example ${ }^{61-63}$

In stark contrast, although weak CT asymmetry was evident by age 4, we observed considerable developmental growth and lifespan change in CT asymmetry thereafter. Developmental trajectories showed non-linear asymmetry growth by virtue of accelerated thinning of the non-dominant hemisphere, and led to maximally established asymmetry around $\sim 25$ years of age. These trajectories clearly suggest differentiation of the cortex is occurring with respect to CT asymmetry in development, possibly, though not necessarily, suggesting CT asymmetry may be more amenable to experiencedependent plastic change. Still, as cortical thinning in childhood is thought to partly reflect likely learning-dependent processes such as intracortical myelination ${ }^{64}$ and possibly pruning of initially overproduced synapses ${ }^{65,66}$ and neuropil reduction, CT asymmetry may reflect hemispheric differences in the developmental optimization of cortical networks at least partly shaped by childhood experience. This raises the possibility CT asymmetry may be a marker of ontogenetic hemispheric specialization within neurocognitive networks. Our findings in development agree with work finding a similar left-right CT asymmetry pattern shows rapid asymmetry increase in the first years of life ${ }^{11}$, with especially rapid increase in leftward mPFC ${ }^{11}$. As we also observed rapid differentiation in mPFC that spanned across childhood and adolescence (Fig 2C-D; SI Fig. 7), we extend these earlier findings in neonates ${ }^{11}$. As prefrontal CT asymmetry seems particularly vulnerable in neurodevelopmental disorders ${ }^{21}$, aging, and Alzheimer's disease ${ }^{10}$, these trajectories may provide a useful normative reference. With regards to aging, most clusters exhibited the expected aging-associated reduction of CT asymmetry we have previously shown is a feature of aging in heteromodal cortex ${ }^{10}$. The differentiation and dedifferentiation of CT asymmetry at either end of life we show here underscores its proposed role in supporting optimal brain organization and function.

For SA asymmetry, we uncovered a covariance structure that almost perfectly replicated across datasets. In general, this fit with a multifaceted view ${ }^{27,34,35}$, in which most asymmetries were either not or only weakly correlated, but reliably so. However, we identified several regions wherein SA asymmetry reliably correlated within individuals, showing the variance in cortical asymmetries is not always dissociable, as often thought $27,34,35$. The strongest relationships all pertained to asymmetries that were proximal in cortex but opposite in direction. Several of these were underpinned by high asymmetryasymmetry genetic correlations, illustrating cerebral lateralizations in SA that are formed under common geneticdevelopmental influence, and in agreement with likely prenatal origins for SA asymmetry ${ }^{26,60}$.

For CT asymmetry, we also uncovered a common covariance structure - particularly clear in UKB - that nevertheless replicated with moderate precision across datasets. Furthermore, a single global factor explained a relatively high proportion of variance in CT asymmetry in UKB, and a strong correlation across 38,172 individuals further suggested CT asymmetry is globally interrelated across the cortex (Fig. 3D). These data for CT indicate individuals tend towards either leftward asymmetry, rightward asymmetry, or symmetry, both globally across the cortex and irrespective of the regionspecific average direction of asymmetry (SI Figs. 9-11). This result seems in broad agreement with the notion that some lateralized genetic-developmental programs may trigger lateralization in either direction ${ }^{35}$ or lose their directional bias through environmental interaction ${ }^{35}$. As CT asymmetry seems established at but minimal from birth ${ }^{11}$, genetic effects may determine the average region-specific hemispheric bias in the population, but developmental change may subsequently confer major increases upon its directional variance ${ }^{35}$. Overall, the evidence converges to suggest a high degree of developmental change may shape CT asymmetry and lead to higher directional variability in the population. Thus, far from being independent phenotypes ${ }^{27,34}$, CT asymmetries may be globally interrelated across the cortex and their direction coordinated through development. 
bioRxiv preprint doi: https://doi.org/10.1101/2021.11.25.469988; this version posted December 8, 2021. The copyright holder for this preprint (which was not certified by peer review) is the author/funder, who has granted bioRxiv a license to display the preprint in perpetuity. It is made available under aCC-BY-NC 4.0 International license.

For SA asymmetry, we found replicable patterns of moderate heritability across datasets and across twin and genomic methods. We also found SA asymmetry in the anterior insula is, to our knowledge, the most heritable asymmetry yet reported with genomic methods ${ }^{31,67,68}$, with common SNPs explaining $\sim 19 \%$ variance. This is a substantial improvement on our recent report of $<5 \%{ }^{24}$, and illustrates a benefit of our data-driven population-mapping approach. As we reported recently ${ }^{24}$, we confirm asymmetry in this region associates with handedness (see below). Furthermore, highest SNPheritability for SA was found in all regions that constitute the earliest emerging cortical asymmetries in utero ${ }^{51,69-71}$ : anterior insula, STS, PT, medial occipital cortex, and parahippocampal gyrus (Fig. 5A). Still, we found most SA asymmetries exhibited significant - albeit often lower - heritability, as did most parcels when estimated cortex-wide, and significant heritability was also evident in regions not found to show strong SA asymmetry, such as Broca's area. These effects agree with and elaborate on two genetic explorations using atlas-based methods ${ }^{7,31}$ and reports of heritable SA asymmetry in handedness-associated clusters ${ }^{24}$. By contrast, CT asymmetry was generally not heritable, or showed low and localized heritability effects. We also observed divergent results using twin and genomic methods for CT, possibly due to low-power for twin-models, though we note the SNP-based effects we observed were somewhat in agreement with a previous twin study ${ }^{7}$. Overall, these reproducible results can guide phenotypic selection in future genomic and biological studies on cerebral laterality.

Considered together, lifespan stability possibly from birth ${ }^{26}$, less interindividual directional variability, higher heritability, and phenotypic and genetic correlations all converge to suggest comparatively higher genetic influence upon SA asymmetry and possibly limited plasticity. This agrees with work showing genetic variants associated with (mostly SA) asymmetry are primarily expressed in prenatal life ${ }^{31}$. By contrast, developmental change, high interindividual directional variability and low heritability for CT asymmetry may fit a scenario whereby CT asymmetry may be more responsive to postnatal individual exposures ${ }^{58}$, or driven by random developmental influences ${ }^{72}$. Whether region-specific CT asymmetry-change relates to the maturation of lateralized brain functions ${ }^{72,73}$ will be an important question for future research. Regardless, our results support a relative prenatal-postnatal developmental dichotomy for SA and CT asymmetry.

Screening asymmetries for association with cognition revealed one region - SMG/perisylvian - wherein higher leftward asymmetry related to higher cognition. Across all samples tested, this cluster was consistently the most lateralized, with $\sim 95 \%$ directional concordance (SI Fig. 3), suggesting highly regulated genetic-developmental programs shape its laterality in humans. Asymmetry here is likely related to brain torque ${ }^{25,40}$, a gross anatomical twist of the hemispheres leading to interhemispheric anatomical differences especially around the Sylvian fissure ${ }^{25}$. Given that brain torque also represents a population-level norm ${ }^{74}$, this result suggests disruptions in prenatal cerebral lateralization may lead to cognitive deficits detectable in later life, and agrees with recent work suggesting brain torque may be related to cognitive outcomes ${ }^{41,74}$. That this was found specifically in the most lateralized SA cluster may agree with work suggesting general cognitive abilities that are intra-individually stable across life ${ }^{75,76}$ relate primarily to SA phenotypes that depend mostly on prenatal factors 56,58

Consistent with our recent vertex-wise analysis in UKB ${ }^{24}$, we confirmed leftward SA asymmetry of the anterior insula, and leftward CT asymmetry of somatosensory cortex, is subtly reduced in left handers. Sha et al. ${ }^{24}$ reported shared genetic influences upon handedness and cortical asymmetry in anterior insula and other more focal regions not identified with the approach used here. Anterior insula lies within a left-lateralized functional language network ${ }^{77}$, and its structural asymmetry may relate to language lateralization ${ }^{33,78,79}$ in which left-handers show increased incidence of atypicality $45,80,81$. Together with observations that its asymmetry emerges early in utero ${ }^{69}$, we agree with others ${ }^{33}$ that future research will find this ontogenetically foundational region of cortex ${ }^{82,83}$ a fruitful line of inquiry for understanding genetic-developmental mechanisms influencing laterality phenotypes. Leftward CT asymmetry reduction in somatosensory cortex in left handers also echoes our recent report, where it was suggested to reflect plastic adaptation to an already-established hand preference ${ }^{24}$. We extend these results by showing CT asymmetry both postcentrally and in general shows developmental differentiation and lifespan change. Given this region overlaps with functional representations of the hands ${ }^{54,55,84}-$ as in Sha et al. ${ }^{24}$ - and our approach also detected no significant heritability, these findings may also fit a scenario whereby CT asymmetries are amenable to alteration through use-dependent plasticity and possibly carry information regarding grouplevel hemispheric specializations of function. However, the small effects cast doubt on the utility of cortical asymmetry to predict individual hand preference.

Asymmetry-relationships with other factors were often compatible with those reported in the ENIGMA meta-analysis ${ }^{7}$. Concerning sex effects - which were small and differing in direction - we similarly observed leftward SA asymmetry in temporal and SMG/perisylvian regions to be larger in males ${ }^{7}$, replicating earlier findings ${ }^{32}$. Previous genetic analyses imply steroid-hormone pathways underly this difference ${ }^{32}$, and sex in general was found to be more predictive than ICV both here and elsewhere ${ }^{32}$. We also found lower SA asymmetry in medial prefrontal cortex in males that was compatible with this earlier report ${ }^{7}$. Inconsistencies evident between ours and the ENIGMA report include findings of increased (here) and decreased ${ }^{7}$ lateral parietal SA asymmetry in males, and increased ${ }^{7}$ and decreased (here) entorhinal CT asymmetry in males, and our approach detected other regions slightly more asymmetric in males (e.g. STS). Possibly, differences in sample median age (here UKB $=\sim 64$; Kong et al. $=26^{7}$ ) and potential sex-differences in age decline trajectories ${ }^{85}$ may underlie some inconsistencies, possibly moreso for CT measures in structures vulnerable to age-related degeneration ${ }^{24}$.

Several limitations should be mentioned. First, our delineation of population-level asymmetry used a single analysis software, and it is unclear to what extent differences in pipelines account for mixed results ${ }^{7,8,18-20,10-17}$, though several studies suggest our results reproduce across analysis systems ${ }^{11,12,20,23}$. Second, while GAMMs are considered an optimal 
bioRxiv preprint doi: https://doi.org/10.1101/2021.11.25.469988; this version posted December 8, 2021. The copyright holder for this preprint (which was not certified by peer review) is the author/funder, who has granted bioRxiv a license to display the preprint in perpetuity. It is made available under aCC-BY-NC 4.0 International license.

modelling technique for longitudinal lifespan data and are robust to non-normal age distributions ${ }^{86}$, relative underrepresentation of the mid-adulthood age-range may drive trajectory inflection points around this age ${ }^{10}$, suggesting caution is warranted regarding interpreting mid-life inflection points as reflecting real change. Third, though the differing heritability methods applied enabled replication for SA, twin studies are prone to overestimating heritability due to unmet assumptions ${ }^{87}$, whereas SNP-based methods may not capture all phenotype-relevant genetic variance and have their own assumptions ${ }^{88}$. Indeed, we found twin-based estimates were often substantially higher even where only nominally significant, agreeing with recent calls for caution when interpreting twin-based heritability estimates ${ }^{87}$. Fourth, we imposed a necessary cluster size limit for overlapping asymmetry effects across samples, and thus more focal asymmetries may also be informative in relation to the factors tested here ${ }^{24}$. Fifth, as only dichotomous handedness self-reports are available with UKB, future studies might benefit from incorporating more nuanced handedness assessments not currently available in data of this size. Relatedly, because UKB cognitive data is not exhaustive (e.g. fluid IQ ranges from 1-13), we extracted the common variance across core tests to index general cognitive ability. This approach did not permit testing associations with specific cognitive abilities, which may be highly informative in the context of asymmetry, particularly in the case of lateralized cognition ${ }^{89}$.

Overall, we provide an openly-available comprehensive characterization of asymmetry in the cerebral cortex including longitudinal lifespan changes, heritability, and individual differences that bears enough reproducibility to be used as a standard in future research.

\section{Methods}

\subsection{Samples}

We used anatomical T1 -weighted (T1w) scans from 7 independent international MRI datasets originating from 4 countries (see SI Table 1 for an overview of samples used for each analysis).

\subsubsection{Reproducibility across samples}

To delineate average adult patterns of whole-cortical SA and CT asymmetry, we restricted the age-range of all samples used in the vertex-wise analyses to 18-55. Dataset 1: Here, the Center for Lifespan Changes in Brain and Cognition $(\angle C B C)$ sample comprised 1572 mixed cross-sectional and longitudinal scans ( $\mathrm{N}$ longitudinal $=812$; timepoint range $=1$ 6) from 923 participants (mean age $=30.6 \pm 9.6$ ) collected across 2 scanners. Additionally, 125 individuals were doublescanned at the same timepoint on both scanners. Dataset 2: The Cambridge Centre for Ageing and Neuroscience (Cam$C A N)^{90}$ sample comprised cross-sectional scans of 321 individuals (mean age $\left.=38.7 \pm 9.7\right)^{91}$. Dataset 3: The Dallas Lifespan Brain Study (DLBS) ${ }^{92}$ sample comprised cross-sectional scans of 160 individuals (mean age $=37.5 \pm 10.7$ ). Dataset 4: The Southwest University Adult Lifespan Dataset (SALD) ${ }^{93}$ sample comprised cross-sectional scans of 301 individuals (mean age $=33.7 \pm 11.5$ ). Dataset 5: The $I X I$ sample comprised cross-sectional scans of 313 healthy individuals collected across 3 scanners (mean age $=36.8 \pm 9.6$; http://brain-development.org/ixi-dataset). Dataset 6: Here, the Human Connectome Project $(H C P) 1200{ }^{94}$ sample comprised 1111 scans (mean age $=28.8 \pm 3.7$ ). Dataset 7: Here, the UKB sample consisted of 1000 randomly sampled cross-sectional scans (mean age $=52.1 \pm 1.9$ ), restricted to be comparable in size to the other datasets in this analysis.

\subsubsection{Lifespan trajectories}

Here, we used the full age-range of LCBC (4.1 - 89.4 years), with a sample comprising 3937 cross-sectional and longitudinal scans (N longitudinal = 2762) from 1886 individuals (females $=1139$; mean age $=36.8$ ) collected across 4 scanners (271 double-scans) ${ }^{95,96}$.

\subsubsection{Interregional correlations}

Here, we used the three largest datasets; LCBC ( $N=923 ; \mathrm{N}$ obs = 1572), HCP (N=1109; SI Fig. 8) and UKB (N=38,172).

\subsubsection{Heritability and individual differences}

To assess heritability, we used HCP 1200 extended twin data (SI Methods), and estimated SNP-heritability using the UKB imaging sample with genome-wide data $(N=31,433$; see 2.2.5). For individual differences analyses, we used the UKB imaging sample with the maximum number of available observations for each variable-of-interest (see 2.2.6).

\subsection{MRI preprocessing}

T1w anatomical images (see SI Table 3 for MRI acquisition parameters) were processed with FreeSurfer (v6.0.0) ${ }^{97}$ and vertex-wise SA and CT morphometry estimates were obtained for each MRI observation (SI Methods). SA and CT maps of the $\mathrm{LH}$ and $\mathrm{RH}$ of each participant in each dataset were resampled from the native cortical geometry to a symmetrical surface template ("LH_sym") ${ }^{14,98}$ based on cross-hemispheric registration ${ }^{99}$. This procedure achieves vertex-wise alignment of the data from each participant and homotopic hemisphere in a common analysis space. We then applied an $8 \mathrm{~mm}$ FWHM Gaussian kernel to surface-smooth the LH and RH data.

\subsection{Data analysis}

\subsubsection{Reproducibility across samples: population-level asymmetry}

We assessed SA and CT asymmetry vertex-wise using FreeSurfer's Linear Mixed Effects (LME) tool ${ }^{100}$. Asymmetry was delineated via the main effect of Hemisphere (controlling for Age, Age $\times$ Hemisphere, Sex, Scanner [where applicable], with a random subject term). For each sample and metric, we computed mean Asymmetry Index maps (Al; defined as (LH$\mathrm{RH}) /((\mathrm{LH}+\mathrm{RH}) / 2))$. Spatial overlap of Al maps across datasets was quantified using Pearson's $r$. To delineate regions 
bioRxiv preprint doi: https://doi.org/10.1101/2021.11.25.469988; this version posted December 8, 2021. The copyright holder for this preprint (which was not certified by peer review) is the author/funder, who has granted bioRxiv a license to display the preprint in perpetuity. It is made available under aCC-BY-NC 4.0 International license.

exhibiting robust SA and CT asymmetry across datasets, we thresholded and binarized the Al maps by a given absolute effect size (SA = 5\%; CT = 1\%; achieving $p[F D R]<.001$ in most datasets with FreeSurfer's 2-stage FDR-procedure ${ }^{100}$ ), and summed the binary maps. After removing the smallest clusters $\left(<200 \mathrm{~mm}^{2}\right)$, a set of robust clusters was defined as those exhibiting overlapping effects in 6 out of 7 samples. We then extracted SA and CT data in symmetrical space for each cluster, subject, and hemisphere, spatially averaging across vertices.

\subsubsection{Lifespan trajectories}

Factor-smooth GAMMs ("gamm4" ${ }^{101}$ ) were used to fit a smooth Age trajectory per Hemisphere, and assess the smooth Age $\times$ Hemisphere interaction in our clusters (SI Methods).

\subsubsection{Interregional correlations}

We assessed covariance matrices between asymmetries and their replication, separately for SA and CT (SI Methods). We also post-hoc tested whether covariance between asymmetries was related to proximity in cortex, and whether observed covariance patterns for CT reflected a global factor (SI Methods).

\subsubsection{Heritability}

Heritability of SA and CT asymmetry was assessed using both twin- and SNP-based methods, both for our set of robust clusters and cortex-wide across 500 parcels ${ }^{50}$. For cluster analyses, significance was considered at Bonferroni-corrected $\mathrm{p}<.05$ applied separately across each metric. Cortex-wide significance was considered at $p(F D R)<.05$ (500 tests per map). Twin heritability for each Al was assessed using ACE models in "OpenMx" ${ }^{103}$ (SI Methods). For SNP-heritability, the genetic sample consisted of 31,433 UKB participants (application \#32048) with imaging and quality checked genetic data $(654,584$ SNPs; SI Methods).

\subsubsection{Associations with Cognition, Sex, Handedness, \& ICV}

Finally, we assessed relationships between asymmetry in our robust clusters and cognitive ability, handedness, sex and ICV (SI Methods). Significance was considered at Bonferroni-corrected $\alpha=p<7.3^{-5}(.01 / 136$ [34 clusters $\left.\times 4]\right)$.

\section{Data sharing/availability}

All summary-level maps are available at neurovault.org/XXXX (upon acceptance), and maps, shareable data and code are available at https://github.com/jamesmroe/PopAsym. All datasets used in this work are openly available, with the exception of LCBC, where participants have not consented to publicly share data. DLBS, SALD, and IXI are available without restrictions (https://fcon_1000.projects.nitrc.org/indi/retro/dlbs.html; CC BY-NC;

http://fcon_1000.projects.nitrc.org/indi/retro/sald.html; CC BY-NC; https://brain-development.org/ixi-dataset; CC BY-SA 3.0). Accordingly, we have made the individual-level maps for these samples available and our code can be used to reproduce vertex-wise analyses in these samples. As differing restrictions apply to the remaining samples (Cam-CAN, HCP; UKB), requests for data access must be submitted and approved via the relevant channel.

\section{Acknowledgements}

Scripts were ran on the Colussus processing cluster at the University of Oslo, and on resources provided by UNINETT Sigma2 (NN9769K). LCBC funding: European Research Council under grants 283634, 725025 (to A.M.F.), and 313440 (to K.B.W.); Norwegian Research Council (to A.M.F. and K.B.W.) under grants 249931 (TOPPFORSK) and 302854 (FRIPRO; to Y.W.), The National Association for Public Health's dementia research program, Norway (to A.M.F). Some of the data used in the preparation of this work were obtained from the MGH-USC Human Connectome Project (https://ida.loni.usc.edu/login.jsp).

\subsection{References}

1. van Kesteren, E.-J. \& Kievit, R. A. Exploratory factor analysis with structured residuals for brain network data. Netw. Neurosci. 1-45 (2020). doi:10.1162/netn a 00162

2. Stark, D. E. et al. Regional Variation in Interhemispheric Coordination of Intrinsic Hemodynamic Fluctuations. J. Neurosci. 28, 13754-13764 (2008).

3. Schmitt, J. E., Giedd, J. N., Raznahan, A. \& Neale, M. C. The genetic contributions to maturational coupling in the human cerebrum: A longitudinal pediatric twin imaging study. Cereb. Cortex 28, 3184-3191 (2018).

4. Chen, C. H. et al. Genetic topography of brain morphology. Proc. Natl. Acad. Sci. U. S. A. 110, 17089-17094 (2013).

5. Eyler, L. T. et al. Conceptual and Data-based Investigation of Genetic Influences and Brain Asymmetry: A Twin Study of Multiple Structural Phenotypes. J. Cogn. Neurosci. 26, 1100-1117 (2014).

6. Raznahan, A. et al. Patterns of coordinated anatomical change in human cortical development: A longitudinal neuroimaging study of maturational coupling. Neuron 72, 873-884 (2011).

7. Kong, X.-Z. et al. Mapping cortical brain asymmetry in 17,141 healthy individuals worldwide via the ENIGMA Consortium. Proc. Natl. Acad. Sci. 115, E5154-E5163 (2018).

8. Chiarello, C., Vazquez, D., Felton, A. \& McDowell, A. Structural asymmetry of the human cerebral cortex: Regional and between-subject variability of surface area, cortical thickness, and local gyrification. Neuropsychologia 93, 365-379 (2016).

9. Meyer, M., Liem, F., Hirsiger, S., Jäncke, L. \& Hänggi, J. Cortical surface area and cortical thickness demonstrate 
bioRxiv preprint doi: https://doi.org/10.1101/2021.11.25.469988; this version posted December 8, 2021. The copyright holder for this preprint (which was not certified by peer review) is the author/funder, who has granted bioRxiv a license to display the preprint in perpetuity. It is made available under aCC-BY-NC 4.0 International license.

10.

11.

13.

14.

15.

16.

17

18.

19.

20.

21.

22.

23.

24.

25.

26.

27.

28.

29.

30.

31.

32.

33.

34.

35.

36.

37.

38.

39.

40.

41.

42.

43.

44.

45.

46.

47.

48.

differential structural asymmetry in auditory-related areas of the human cortex. Cereb. Cortex 24, 2541-2552 (2014). Roe, J. M. et al. Asymmetric thinning of the cerebral cortex across the adult lifespan is accelerated in Alzheimer's disease. Nat. Commun. 12, 1-11 (2021).

Li, G., Lin, W., Gilmore, J. H. \& Shen, D. Spatial patterns, longitudinal development, and hemispheric asymmetries of cortical thickness in infants from birth to 2 years of age. J. Neurosci. 35, 9150-9162 (2015).

Luders, E. et al. Hemispheric asymmetries in cortical thickness. Cereb. Cortex 16, 1232-1238 (2006).

Zhou, D., Lebel, C., Evans, A. \& Beaulieu, C. Cortical thickness asymmetry from childhood to older adulthood.

Neuroimage 83, 66-74 (2013).

Maingault, S., Tzourio-Mazoyer, N., Mazoyer, B. \& Crivello, F. Regional correlations between cortical thickness and surface area asymmetries: A surface-based morphometry study of 250 adults. Neuropsychologia 93, 350-364 (2015). Shaw, P., Lalonde, F. \& Al, L. C. et. Development of Cortical Asymmetry in Typically Developing Children and Its Disruption in Attention-Deficit/Hyperactivity Disorder. 66, 888-896 (2009).

Lou, Y. et al. Brain asymmetry differences between Chinese and Caucasian populations : a surface-based morphometric comparison study. (2019).

18, 1427-1431 (2007).

Zhou, D. et al. Preserved cortical asymmetry despite thinner cortex in children and adolescents with prenatal alcohol exposure and associated conditions. Hum. Brain Mapp. 39, 72-88 (2018).

Koelkebeck, K. et al. The contribution of cortical thickness and surface area to gray matter asymmetries in the healthy human brain. Hum. Brain Mapp. 35, 6011-6022 (2014).

Plessen, K. J., Hugdahl, K., Bansal, R., Hao, X. \& Peterson, B. S. Sex, age, and cognitive correlates of asymmetries in thickness of the cortical mantle across the life span. J Neurosci 34, 6294-6302 (2014).

Postema, M. C. et al. Altered structural brain asymmetry in autism spectrum disorder in a study of 54 datasets. Nat. Commun. 10, 1-12 (2019).

Thompson, P. M. et al. Tracking Alzheimer's disease. Ann. N. Y. Acad. Sci. 1097, 183-214 (2007).

Lyttelton, O. C. et al. Positional and surface area asymmetry of the human cerebral cortex. Neuroimage 46, 895-903 (2009).

Sha, Z. et al. Handedness and its genetic influences are associated with structural asymmetries of the cerebral cortex in 31,864 individuals. Proc. Natl. Acad. Sci. 118, 1-9 (2021).

Toga, a W. \& Thompson, P. M. Mapping brain asymmetry. Nat. Rev. Neurosci. 4, 37-48 (2003).

$\mathrm{Li}, \mathrm{G}$. et al. Mapping longitudinal hemispheric structural asymmetries of the human cerebral cortex from birth to 2 years of age. Cereb. Cortex 24, 1289-1300 (2014).

Bain, J. S., Filo, S. \& Mezer, A. A. The robust and independent nature of structural STS asymmetries. Brain Struct Funct. 224, 3171-3182 (2019) Neuroimage 153, 246-261 (2017).

Nie, J., Li, G. \& Shen, D. Development of cortical anatomical properties from early childhood to early adulthood. Neuroimage 76, 216-224 (2013).

Li, G. et al. Mapping region-specific longitudinal cortical surface expansion from birth to 2 years of age. Cereb. Cortex 23, 2724-2733 (2013).

Sha, Z. et al. The genetic architecture of structural left-right asymmetry of the human brain. Nat. Hum. Behav. (2021). doi:10.1038/s41562-021-01069-w

Guadalupe, T. et al. Asymmetry within and around the human planum temporale is sexually dimorphic and influenced by genes involved in steroid hormone receptor activity. Cortex 62, 41-55 (2015).

Chiarello, C., Vazquez, D., Felton, A. \& Leonard, C. M. Structural asymmetry of anterior insula: Behavioral correlates and individual differences. Brain Lang. 126, 109-122 (2013).

Rentería, M. E. Cerebral asymmetry: A quantitative, multifactorial, and plastic brain phenotype. Twin Res. Hum. Genet. 15, 401-413 (2012).

Francks, C. Exploring human brain lateralization with molecular genetics and genomics. Ann. N. Y. Acad. Sci. 1359, 113 (2015).

Liu, H., Stufflebeam, S. M., Sepulcre, J., Hedden, T. \& Buckner, R. L. Evidence from intrinsic activity that asymmetry of the human brain is controlled by multiple factors. Proc. Natl. Acad. Sci. U. S. A. 106, 20499-20503 (2009).

point of hemispheric indecision. Neuropsychologia 36, 1275-1282 (1998)

Ocklenburg, S., Güntürkün, O., Hugdahl, K. \& Hirnstein, M. Laterality and mental disorders in the postgenomic age - A closer look at schizophrenia and language lateralization. Neurosci. Biobehav. Rev. 59, 100-110 (2015).

Kong, X.-Z. et al. Large-Scale Phenomic and Genomic Analysis of Brain Asymmetrical Skew. Cereb. Cortex 1-18 (2021). doi:10.1093/cercor/bhab075

LeMay, M. Morphological Cerebral Asymmetries of Modern Man, Fossil Man, and Nonhuman Primate. Ann. N. Y. Acad. Sci. 280, 349-366 (1976).

Zhao, L., Matloff, W., Shi, Y., Cabeen, R. P. \& Toga, A. W. Mapping Complex Brain Torque Components and Their Genetic and Phenomic Architecture in 24, 112 healthy individuals. (2021).

Moodie, J. E. et al. Fluctuating asymmetry in brain structure and general intelligence in 73-year-olds. Intelligence 78 101407 (2020)

Yeo, R. A., Ryman, S. G., Pommy, J., Thoma, R. J. \& Jung, R. E. General cognitive ability and fluctuating asymmetry of brain surface area. Intelligence 56, 93-98 (2016).

Kong, X. et al. Mapping brain asymmetry in health and disease through the ENIGMA consortium. Hum. Brain Mapp. 115 (2020).

Wiberg, A. et al. Handedness, language areas and neuropsychiatric diseases : insights from brain imaging and genetics. Brain 1-10 (2019). doi:10.1093/brain/awz257

van der Meer, D. et al. Quantifying the Polygenic Architecture of the Human Cerebral Cortex: Extensive Genetic Overlap between Cortical Thickness and Surface Area. Cereb. Cortex 30, 5597-5603 (2020).

Eyler, L. T. et al. A comparison of heritability maps of cortical surface area and thickness and the influence of adjustment for whole brain measures: A magnetic resonance imaging twin study. Twin Res. Hum. Genet. 15, 304-314 (2012).

Desikan, R. S. et al. An automated labeling system for subdividing the human cerebral cortex on MRI scans into gyral 
bioRxiv preprint doi: https://doi.org/10.1101/2021.11.25.469988; this version posted December 8, 2021. The copyright holder for this preprint (which was not certified by peer review) is the author/funder, who has granted bioRxiv a license to display the preprint in perpetuity. It is made available under aCC-BY-NC 4.0 International license.

Miller, K. L. et al. Multimodal population brain imaging in the UK Biobank prospective epidemiological study. Nat. Neurosci. 19, 1523-1536 (2016).

50. Schaefer, A. et al. Local-Global Parcellation of the Human Cerebral Cortex from Intrinsic Functional Connectivity MRI Cereb. Cortex 1-20 (2017). doi:10.1093/cercor/bhx179

51. Habas, P. A. et al. Early folding patterns and asymmetries of the normal human brain detected from in utero MRI. Cereb. Cortex 22, 13-25 (2012).

52. Glasel, H. et al. A robust cerebral asymmetry in the infant brain: The rightward superior temporal sulcus. Neuroimage 58, 716-723 (2011).

53. Foundas, A. L., Eure, K. F., Luevano, L. F. \& Weinberger, D. R. MRI asymmetries of Broca's area: The pars triangularis and pars opercularis. Brain Lang. 64, 282-296 (1998).

54. Penfield, W. \& Boldrey, E. Somatic Motor and Sensory Representation in Man. Brain 389-443 (1937).

55. Roux, F. E., Djidjeli, I. \& Durand, J. B. Functional architecture of the somatosensory homunculus detected by electrostimulation. J. Physiol. 596, 941-956 (2018).

56. Walhovd, K. B. et al. Neurodevelopmental origins of lifespan changes in brain and cognition. Proc. Natl. Acad. Sci. U. S. A. 113, 9357-9362 (2016).

57. Grasby, K. L. et al. The genetic architecture of the human cerebral cortex. Science (80-. ). 367, (2020).

58. Fjell, A. M. et al. Continuity and Discontinuity in Human Cortical Development and Change From Embryonic Stages to Old Age. Cereb. Cortex 29, 3879-3890 (2019).

59. Wierenga, L. M., Langen, M., Oranje, B. \& Durston, S. Unique developmental trajectories of cortical thickness and surface area. Neuroimage 87, 120-126 (2014).

60. Rakic, P. A small step for the cell, a giant leap for mankind: a hypothesis of neocortical expansion during evolution Trends Neurosci. 18, 383-388 (1995).

61. Chance, S. A., Casanova, M. F., Switala, A. E. \& Crow, T. J. Minicolumnar structure in Heschl's gyrus and planum temporale: Asymmetries in relation to sex and callosal fiber number. Neuroscience 143, 1041-1050 (2006).

62. Tzourio-Mazoyer, N., Crivello, F. \& Mazoyer, B. Is the planum temporale surface area a marker of hemispheric or regional language lateralization? Brain Struct. Funct. 223, 1217-1228 (2018).

63. Ocklenburg, S. et al. Neurite architecture of the planum temporale predicts neurophysiological processing of auditory speech. Sci. Adv. 4, 1-9 (2018).

64. Natu, V. S. et al. Apparent thinning of human visual cortex during childhood is associated with myelination. Proc. Natl. Acad. Sci. 116, 20750-20759 (2019).

65. Petanjek, Z. et al. Extraordinary neoteny of synaptic spines in the human prefrontal cortex. Proc. Natl. Acad. Sci. U. S. A. 108, 13281-13286 (2011).

66. Faust, T. E., Gunner, G. \& Schafer, D. P. Mechanisms governing activity-dependent synaptic pruning in the developing mammalian CNS. Nat. Rev. Neurosci. 22, 657-673 (2021).

67. Cuellar-Partida, G. et al. Genome-wide association study identifies 48 common genetic variants associated with handedness. Nat. Hum. Behav. (2020). doi:10.1038/s41562-020-00956-y

68. Carrion-Castillo, A. et al. Genetic effects on planum temporale asymmetry and their limited relevance to neurodevelopmental disorders, intelligence or educational attainment. Cortex 124, 137-153 (2020).

69. Dubois, J. et al. Structural asymmetries of perisylvian regions in the preterm newborn. Neuroimage 52, 32-42 (2010).

70. Kasprian, G. et al. The prenatal origin of hemispheric asymmetry: An in utero neuroimaging study. Cereb. Cortex 21, 1076-1083 (2011).

71. Hill, J. et al. A Surface-Based Analysis of Hemispheric Asymmetries and Folding of Cerebral Cortex in Term-Born Human Infants. J. Neurosci. 30, 2268-2276 (2010).

72. Bishop, D. V. M. \& Bates, T. C. Heritability of language laterality assessed by functional transcranial Doppler ultrasound: A twin study. Wellcome Open Res. 4, 1-52 (2020)

73. Somers, M. et al. Linkage analysis in a Dutch population isolate shows no major gene for left-handedness or atypical language lateralization. J. Neurosci. 35, 8730-8736 (2015).

74. Kong, X.-Z. et al. Handedness and Other Variables Associated with Human Brain Asymmetrical Skew. bioRxiv 756395 (2019). doi:10.1101/756395

75. Karama, S. et al. Childhood cognitive ability accounts for associations between cognitive ability and brain cortical thickness in old age. Mol. Psychiatry 19, 555-559 (2014).

76. Deary, I. J., Whiteman, M. C., Starr, J. M., Whalley, L. J. \& Fox, H. C. The Impact of Childhood Intelligence on Later Life: Following Up the Scottish Mental Surveys of 1932 and 1947. J. Pers. Soc. Psychol. 86, 130-147 (2004).

77. Labache, L. et al. A SENtence Supramodal Areas AtlaS (SENSAAS) based on multiple task-induced activation mapping and graph analysis of intrinsic connectivity in 144 healthy right-handers. Brain Struct. Funct. 224, 859-882 (2019).

78. Biduła, S. P. \& Króliczak, G. Structural asymmetry of the insula is linked to the lateralization of gesture and language. Eur. J. Neurosci. 41, 1438-1447 (2015).

79. Keller, S. S. et al. Can the language-dominant hemisphere be predicted by brain anatomy? J. Cogn. Neurosci. 23, 2013-2029 (2011).

80. Mazoyer, B. et al. Gaussian mixture modeling of hemispheric lateralization for language in a large sample of healthy individuals balanced for handedness. PLoS One 9, 9-14 (2014).

81. Knecht, S. et al. Handedness and hemispheric language dominance in healthy humans. Brain 123, 2512-2518 (2000)

82. Afif, A., Bouvier, R., Buenerd, A., Trouillas, J. \& Mertens, P. Development of the human fetal insular cortex: Study of the gyration from 13 to 28 gestational weeks. Brain Struct. Funct. 212, 335-346 (2007).

83. Kalani, M. Y. S., Kalani, M. A., Gwinn, R., Keogh, B. \& Tse, V. C. K. Embryological development of the human insula and its implications for the spread and resection of insular gliomas. Neurosurg. Focus 27, 1-4 (2009).

84. Saadon-Grosman, N., Loewenstein, Y. \& Arzy, S. The 'creatures' of the human cortical somatosensory system. Brain Commun. 2, 1-10 (2020).

85. McCarrey, A. C., An, Y., Kitner-Triolo, M. H., Ferrucci, L. \& Resnick, S. M. Sex differences in cognitive trajectories in clinically normal older adults. Psychol. Aging 31, 166-175 (2016).

86. Sørensen, Ø., Walhovd, K. B. \& Fjell, A. M. A Recipe for Accurate Estimation of Lifespan Brain Trajectories, Distinguishing Longitudinal and Cohort Effects. (2020).

87. Dalmaijer, E. S. Twin studies with unmet assumptions are biased towards genetic heritability. bioRxiv 2020.08.27.270801 (2020) 
bioRxiv preprint doi: https://doi.org/10.1101/2021.11.25.469988; this version posted December 8, 2021. The copyright holder for this preprint (which was not certified by peer review) is the author/funder, who has granted bioRxiv a license to display the preprint in perpetuity. It is made available under aCC-BY-NC 4.0 International license.

88. Hibar, D. P. et al. Novel genetic loci associated with hippocampal volume. Nat. Commun. 8, (2017)

89. Ocklenburg, S., Hirnstein, M., Beste, C. \& Güntürkün, O. Lateralization and cognitive systems. Washington, DC APA, Guidel. Dev. Panel Treat. Posttraumatic Stress Disord. Adults. 5, (2014).

90. Shafto, M. A. et al. The Cambridge Centre for Ageing and Neuroscience (Cam-CAN) study protocol: A cross-sectional, lifespan, multidisciplinary examination of healthy cognitive ageing. BMC Neurol. 14, 1-25 (2014).

91. Taylor, J. R. et al. The Cambridge Centre for Ageing and Neuroscience (Cam-CAN) data repository: Structural and functional MRI, MEG, and cognitive data from a cross-sectional adult lifespan sample. Neuroimage 144, 262-269 (2017).

92. Kennedy, K. M. et al. Effects of beta-amyloid accumulation on neural function during encoding across the adult lifespan. Neuroimage 62, 1-8 (2012).

93. Wei, D. et al. Data Descriptor: Structural and functional brain scans from the cross-sectional Southwest University adult lifespan dataset. Sci. Data 5, 1-10 (2018).

94. Van Essen, D. C. et al. The WU-Minn Human Connectome Project: An overview. Neuroimage 80, 62-79 (2013).

95. Fjell, A. M. et al. Self-reported sleep relates to hippocampal atrophy across the adult lifespan - results from the Lifebrain consortium. Sleep 1-15 (2019). doi:10.1093/sleep/zsz280

96. Vidal-Pineiro, D. et al. Cellular correlates of cortical thinning throughout the lifespan. Preprint at https://www.biorxiv.org/content/10.1101/585786v3. (2019).

97. Fischl, B. \& Dale, A. M. Measuring the thickness of the human cerebral cortex from magnetic resonance images. Proc. Natl. Acad. Sci. U. S. A. 97, 11050-5 (2000).

98. Marie, D., Maingault, S., Crivello, F., Mazoyer, B. \& Tzourio-Mazoyer, N. Surface-Based Morphometry of Cortical Thickness and Surface Area Associated with Heschl's Gyri Duplications in 430 Healthy Volunteers. Front. Hum. Neurosci. 10, (2016).

99. Greve, D. et al. A Surface-based Analysis of Language Lateralization and Cortical Asymmetry. J. Cogn. Neurosci. 25, 1477-1492 (2013).

100. Bernal-Rusiel, J. L., Reuter, M., Greve, D. N., Fischl, B. \& Sabuncu, M. R. Spatiotemporal linear mixed effects modeling for the mass-univariate analysis of longitudinal neuroimage data. Neuroimage 81, 358-370 (2013).

101. Wood, S. \& Scheipl, F. gamm4: Generalized Additive Mixed Models using 'mgcv' and 'Ime4'. R package version 0.2-5, available at https://cran.r-project.org/web/packages/gamm4/gamm4.pdf. (2017).

102. Dray, S. \& Dufour, A. B. The ade4 package: Implementing the duality diagram for ecologists. J. Stat. Softw. 22, 1-20 (2007).

103. Neale, M. C. et al. OpenMx 2.0: Extended Structural Equation and Statistical Modeling. Psychometrika 81, 535-549 (2016). 